ORIGINAL STUDY

\title{
The value of posterior rhinomanometry in evaluating patients with snoring and obstructive sleep apnea syndrome
}

\author{
Alex George Stanciu', Alexandra Cirticioiu', Adriana Neagos ${ }^{2}$ \\ ${ }^{1}$ Department of Otorhinolaryngology, Mures County Emergency Hospital, Tirgu Mures, Romania \\ ${ }^{2}$ Department of Otorhinolaryngology, Tirgu Mures University of Medicine and Pharmacy, Romania
}

\begin{abstract}
BACKGROUND. Nowadays, snoring and obstructive sleep apnea are the most common sleep-related breathing disorders (SRBD) found in the medical practice and they are estimated to affect approximately $4 \%$ of men and $2 \%$ of women in the middle-aged workforce. There are some ENT pathologies that are involved in snoring, and also in obstructive sleep apnea syndrome. The nose plays an important part in both breathing and SRBD. The aim of this study was to determine the role of posterior rhinomanometry in the diagnoses of snoring and obstructive sleep apnea syndrome.

MATERIAL AND METHODS. We present a retrospective observational study that took place during a three-year period, between 2009 and 2012. In this study were included 110 subjects, who have undergone polysomnographic investigations, as well as an ENT clinical examination focused on endoscopy and posterior rhinomanometry evaluation.

RESULTS. The mean age was $46.09 \pm 10.91$ years, the mean height was $177.58 \pm 10.02 \mathrm{~cm}$ and the mean weight was $98.45 \pm$ $17.74 \mathrm{~kg}$. Analysing these results, we found a significant correlation between them and the severity of the sleep pathology. The posterior rhinomanometry results were correlated with nasal allergic rhinitis $(p=0.06)$ and not correlated with other nasal pathology, such as nasal septum deviation. The changes in the size and shape of the tongue base or epiglottis proved to have an important impact upon rhinomanometric values, with a statistically significant value $(p=0.03$, respectively $p=0.08)$.
\end{abstract}

CONCLUSION. All diagnostic methods must be interpreted in connection with each other.

KEYWORDS: snoring, obstructive sleep apnea, posterior rhinomanometry, polysomnography, nasal endoscopy

\section{INTRODUCTION}

The most frequent and common respiratory sleep pathology encountered in medical practice is snoring associated or not with obstructive sleep apnea syndrome (OSAS). Obstructive sleep apnea is characterized by repetitive, partial or complete obstruction of the upper airway during sleep despite simultaneous respiratory effort, resulting in episodic hypoxemia and arousal. Respiratory disorders during sleep are very common in the general population and they are estimated to affect approximately $4 \%$ of men and $2 \%$ of women in the middle-aged workforce and laboratory investigations in sleepiness potentially increase day by day.
It is well known that nasal breathing is the physiological one and that the nasal passages, by their complex structure and rich vascular, lymphatic, glandular and nervous formations, fulfil a very important physiological role. The calibre of the nasal passages, by their architectonic and morphofunctional integrity of the mucosa, help regulate the respiratory airflow, through its vascular-erectile function. The nasal mucosa, due to its morphological structure, ensures the inspired air conditioning function by the following three processes: heating, moistening and filtering. The ENT changes responsible for upper airway obstruction can be represented by nasal pathology, with or without oral and hypopharyngeal pathology. Multilevel obstruction is often present in snoring and ob- 
structive sleep apnea. As the nose is the first anatomical boundary of the upper airway, nasal obstruction may contribute to sleep-disordered breathing ${ }^{1}$.

The most commonly encountered nasal pathologies associated with nasal obstruction are: nasal septum deviation, chronic hypertrophic rhinitis, nasal polyposis, adenoids, sinusochoanal polyps, etc. The sinonasal disorders can be accompanied by tonsillar hypertrophy, hypertrophy of the tongue base, soft palate hypertrophy, narrowing of the hypopharynx, epiglottis malformations, tumors and inflammatory laryngeal pathology.

Nocturnal polysomnography is considered to be the gold standard for diagnosing the patients with sleep apnea. It offers data related to the analysis of sleep stages, ventilation, identification of respiratory events and their consequences on heart rhythm and oxygen saturation. Based on the polysomnography, the diagnosis of obstructive sleep apnea syndrome (OSAS) is certified or not.

The literature shows a correlation between nasal resistance in the supine position and chronic snoring ${ }^{2}$. Also, there are epidemiological studies showing that allergic rhinitis affects $9-42 \%$ of the population ${ }^{3}$.

The nasal obstruction can be diagnosed with posterior rhinomanometry in the awake. Many studies show that, in the awake state, nasal obstruction is an

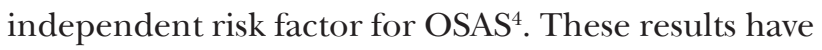
been contradicted by other studies, which found differences between apnea and snoring patients with nasal resistance ${ }^{5}$.

A similar study measuring supine nasal resistance failed to find any correlation with sleep disturbance indices. However, the study did not include patients with significant nasal obstruction, while all measurements were done in non-physiological conditions, with patients having their mouth taped throughout the night to prevent oral breathing ${ }^{6}$.

In this study, we present the Romanian experience in evaluating patients with sleep apnea. We wanted to evaluate the value of posterior rhinomanometry in diagnosing patients with SRBD and also, to show that rhinomanometry associated with polysomnographic and other clinical investigations are very important in evaluating these patients. The flexible pharyngoscopy as investigation is essential to specify the location of the obstruction of the upper airway, especially in cases where the therapeutic attitude involves a surgical procedure.

\section{MATERIAL AND METHODS}

We performed a retrospective observational study during a three-year period, from 2009 to 2012. The study included a number of 110 patients, evaluated in
Tirgu Mures, at Galenus Medical Center. All subjects underwent polysomnographic investigations for diagnosing the obstructive sleep apnea, and only those with chronic snoring and obstructive sleep apnea syndrome have been selected.

Further, in the study were included patients who accomplished the following criteria: they all had personal data, a complete ENT clinical examination, rhinomanometry evaluation, all associated with polysomnographic investigations. The changes in the upper respiratory tract were assessed by rhino-laryngoscopy examination, with particular emphasis on nasal endoscopy, and the results were recorded in the ENT examination form.

From the anamnesis point of view, we were interested in the presence or absence of allergies or nasal breathing disorders. The clinical evaluation focussed on nasal, oro- and hypopharynx anomalies, such as: nasal septum deviation, concha bullosa, inferior turbinate hyperplasia, nasal polyps, nasal pyramid changes, nasal valve insufficiency; hypertrophy of the tonsils and the base tongue; the uvula, aryepiglottic fold and ventricular bands changes; the soft palate mobility in 'A' phonation, the epiglottis form, and also on the mouth opening.

The polysomnography was performed during a period of 6 hours of physiological sleep, not drug-induced sleep. The results have been summarized in the following parameters: apnea - hypopnea index (AHI), oxygen saturation, the number of oxygen desaturations and their degree (ODI), the number of nighttime events (RDI), total sleeping time (TST), sleep efficiency, the number of snoring and the position in which they occur, as well as the sleep stages.

Rhinomanometry was performed using the posterior rhinomanometry program for the evaluation of the nasal resistance.

Endoscopic evaluation of patients, flexible or rigid, was performed during wakefulness and flexible videopharyngoscopy was performed during sleep. By flexible pharyngoscopy we identified the anatomical changes in the pharyngeal wall collapse, in the epiglottis, in the retrovelar, retrolingual and retroepiglottic space. The evaluation was completed using the Mueller maneuver. Selectively, flexible videopharyngoscopy was performed during drug-induced sleep with Propofol $0.5 \mathrm{mg} / \mathrm{kg}$ body - $2 \mathrm{mg} / \mathrm{kg}$ body - the drug-induced sleep endoscopy (DISE).

The study population was divided according to OSAS severity into four groups: AHI $<10$ corresponds to chronic snoring, $10<\mathrm{AHI}<30$ mild OSAS, $30<\mathrm{AHI}$ $<50$ moderate OSAS, and AHI $>50$ defines severe OSAS.

The data were processed in Excel 2007 and the statistical analysis was performed using the MedCalc program, designed for statistics in biomedical research. 
Frequency tables were used to obtain numerical data and percentages. For the univariate analysis of data we used the $\chi 2$ test (dichotomous variables). The differences were considered as having statistical significance when the value of the $p$ parameter was less than 0.05 . The graphical representation was performed using the dispersion diagram (scatter) which, through orientation and dispersion of point clouds, provides an image of the relationship between two variables. Sheer descriptive statistical parameters were calculated for all variables that we considered useful: the medium average, the lowest and highest value for numeric variables, standard deviation $(\mathrm{SD})$, the median, 95\% confidence index (IC 95\%).

\section{RESULTS}

From the total 110 patients that were initially under study, 79 patients were excluded for lack of infor- mation. Thus, in the study group remained a total of 31 patients, aged 24 - 63 years. The gender distribution revealed a predominance of male patients $(27$, representing $87.1 \%$ ) versus female patients $(n=4)$, resulting a statistically significant difference in gender distribution $(p$-value $=0.0001)$. The demographic characteristics of the study subjects are (arithmetic average \pm standard deviation): average age $46.09 \pm$ 10.91 years, average height $177.58 \pm 10.02 \mathrm{~cm}$, average weight $98.45 \pm 17.74 \mathrm{~kg}$, BMI (body mass index) $31.20 \pm 5.09 \mathrm{~kg} / \mathrm{m}^{2}$. Regarding the distribution by age, there was a perfect correlation between age and the frequency of obstructive respiratory pathology (snoring and obstructive sleep apnea), with a $p<0.0001$ (Chart 1).

The average height of the patients was $177.58 \mathrm{~cm}$, with a significant correlation $(p<0.0001)$ between height and sleep respiratory pathology (Chart 2). With an average body weight of $98.45 \pm 17.74 \mathrm{~kg}$ and a $p$-value $<0.0001$, there is a significant influence of

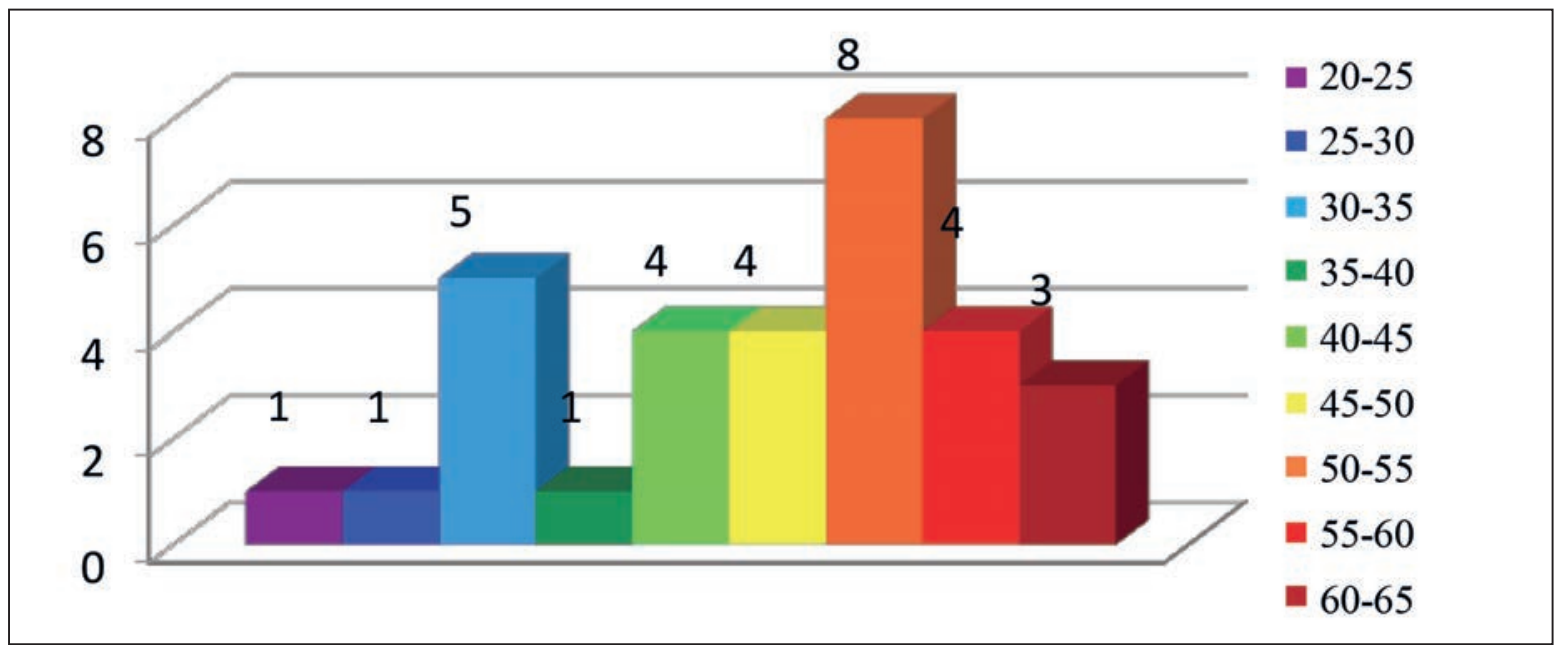

Chart 1 Correlation between age and the frequency of snoring and obstructive sleep apnea

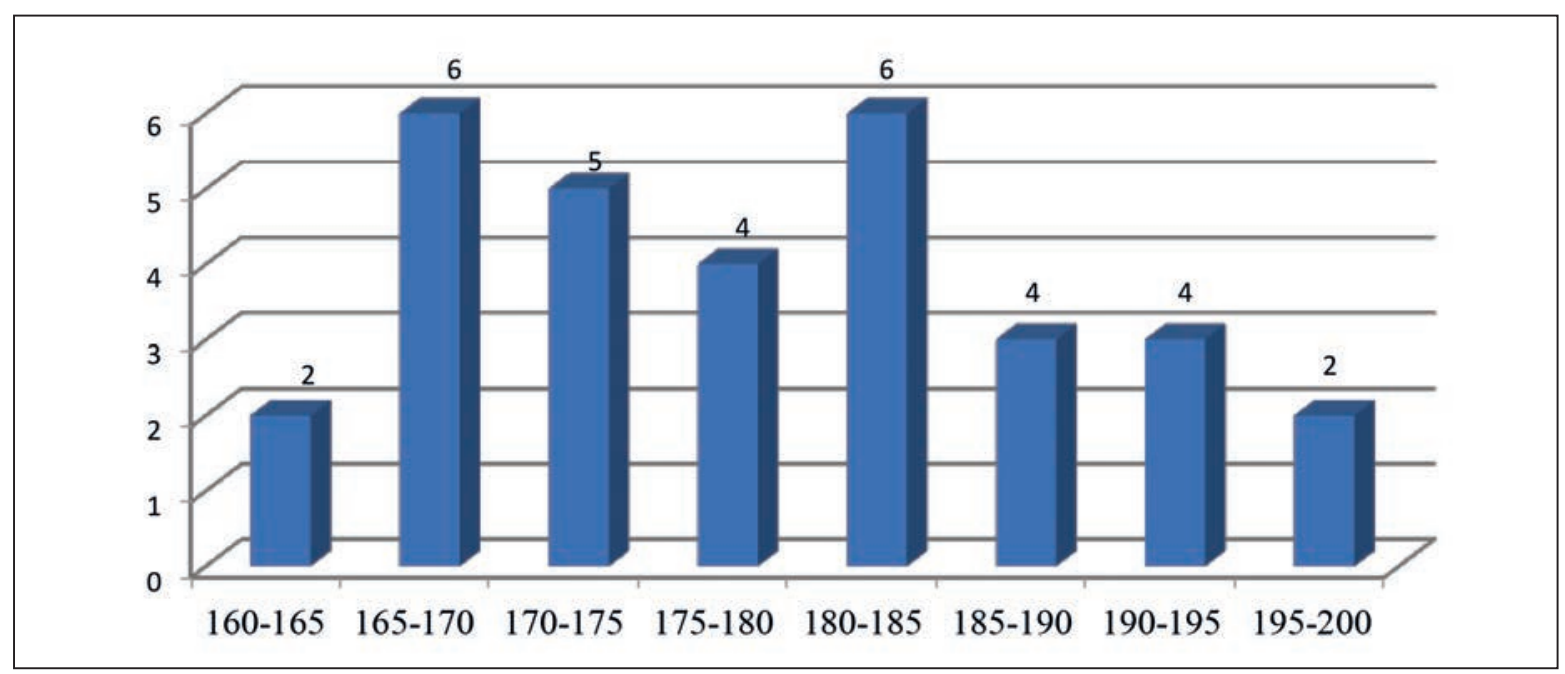

Chart 2 Correlation between patients' height and the frequency of snoring and obstructive sleep apnea 
weight on the frequency of snoring and apnea events (Chart 3).

The AHI mean value was of 21.95. Analysing the snoring flags and the AHI value, we encountered a significant correlation between these two parameters $(p<0.0001)$. Most patients with sleep breathing disorders presented a chronic snoring and mild apnea. Few were the patients with severe OSAS, which made useful the ENT doctor involvement in the treatment of the sleep respiratory pathology (Chart 4).

The average value of the measured sleeping time was $6.10 \pm 1.26$ hours. In one patient $(3.22 \%)$ we have found the total sleeping time under 3 hours, in 3 patients $(9.67 \%)$ between $4-5$ hours, in 4 patients $(12.90 \%)$ between 5-6 hours. The rest of the patients were within the normal values of the sleeping time. This shows a good adaptability of the patients to the somnology lab conditions (Chart 5).

Through nasal endoscopy was demonstrated that the most common anatomical changes were located at the level of the soft palate, uvula and tonsils, throat and nose.

The most common rhinological changes occurring in our study were represented by: nasal deviated septum, inferior turbinate hypertrophy, lateral deviation of the nasal pyramid, associated with nasal obstruction of different degrees. The shape and size of the tonsils (e.g. hypertrophied tonsils), associated or not with a long and/ or wide uvula, thickened mucosal folds, low mobility of the soft palate in phonation "A", elongated soft palate,

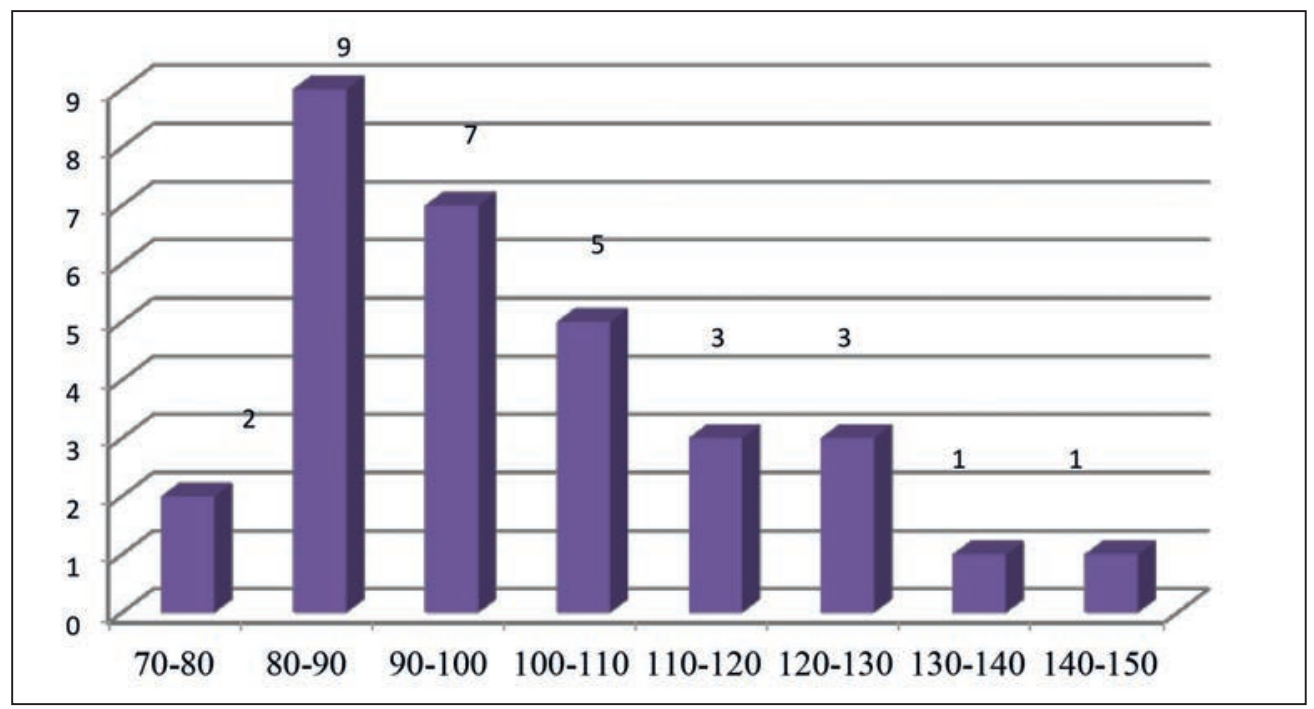

Chart 3 Correlation between body weight, snoring and obstructive sleep apnea

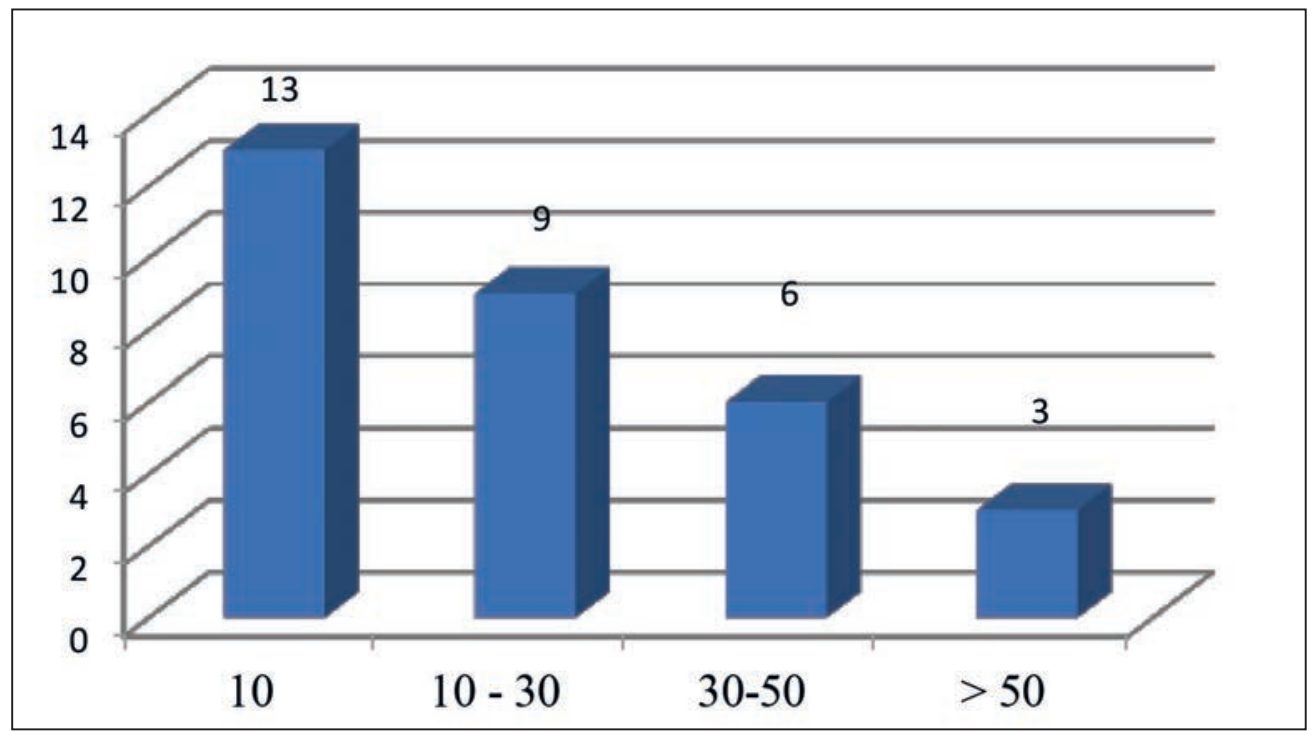

Chart 4 Frequency of snoring and OSAS 


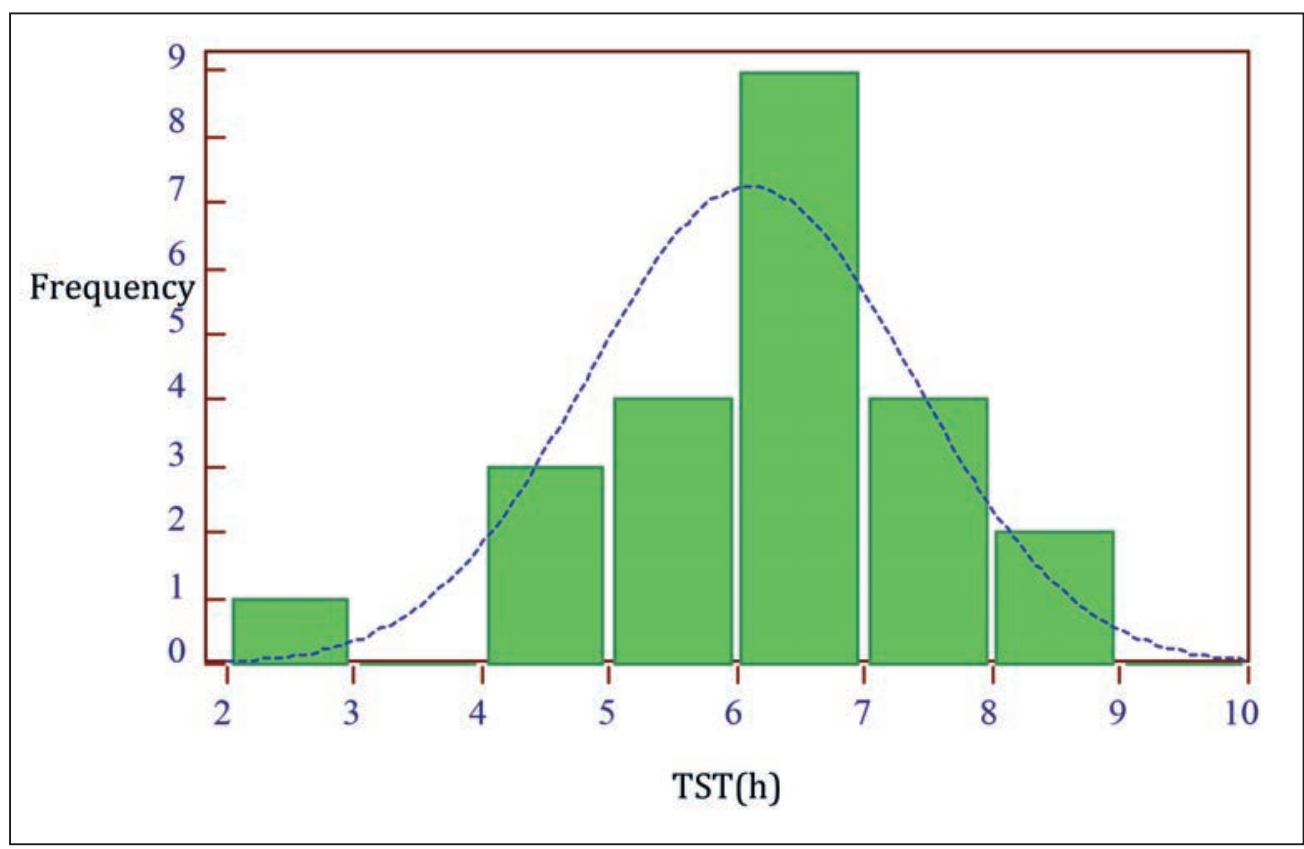

Chart 5 Sleeping time and frequency of snoring and obstructive sleep apnea

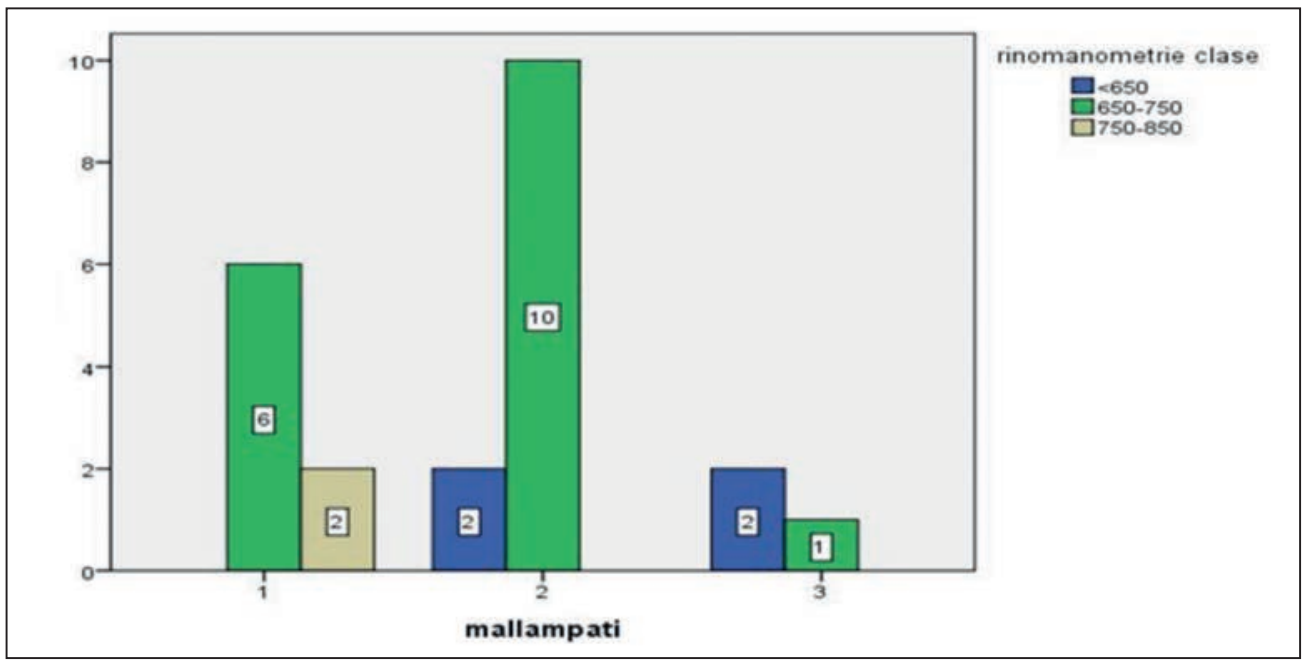

Chart 6 Posterior rhinomanometry and ENT examination results (Mallampati Score between 1 and 3)

tongue base hypertrophy, hypopharyngeal concentric narrowing were other changes responsible for obstructive sleep apnea syndrome in our study group.

Further, we evaluated the correlation between the ENT changes and the apnea-hypopnea index value (AHI). Although the most common nasal pathologies that we have found were inferior turbinate hypertrophy $(p=0.58)$, nasal respiratory disorders $(p=0.98)$, long and/or wider uvula $(p=0.48)$, thickened mucosal folds $(p=0.41)$, they were not considered to be a risk factor in the airflow obstruction of the upper airways in OSAS. Statistically speaking, there was a correlation between nasal pathology such as nasal allergy and the AHI, $p$-value being of 0.06 . As far as the hypopharyngeal and laryngeal pathology is concerned, the epi- glottis shape and tongue base size were correlated with a high AHI $(p=0.05)$.

From the rhinomanometry results we processed the data that were taken from the first measurement, before the surgical correction. Using rhinomanometry classification for assessing nasal obstruction and correlating the anatomical changes of the upper airway with the rhinomanometry results, we found a statistically significant difference $(p=0.03)$ between the size of the base tongue and rhinomanometry values (Chart 6). There was no statistically significant influence of the deviated nasal septum, inferior turbinate hypertrophy or lateral deviated nasal pyramid upon the rhinomanometric measurements ( $p$-value between 0.44 and 0.74 ). 
From all 23 subjects that underwent a polysomnography evaluation, 4 of them had normal rhinomanometric values $(650 \mathrm{ccm} / \mathrm{s}), 17$ had values between $650-750 \mathrm{ccm} / \mathrm{s}$ and 2 of them had values between $750-859 \mathrm{ccm} / \mathrm{s}$. Among the subjects with AHI $<10-$ so those who snore -6 had the nasal flow between $650-$ $750 \mathrm{ccm} / \mathrm{s}, 2$ had nasal air-flow value of $750-850 \mathrm{ccm} / \mathrm{s}$. 2 of the patients with an AHI higher than 50 - with severe OSAS - had a rhinomanometry value of $650-750 \mathrm{ccm} / \mathrm{s}$. In the present study, we found no statistically significant difference between the nasal pathology, according to the posterior rhinomanometry values, and the apnea-hypopnea index $(p=0.19)$.

The relationship between OSAS and rhinomanometric results required a regression analysis and correlation, considering as dependent variable the OSAS, the quantitative one represented by oxygen desaturation, and as an independent variable the rhinomanometric results. We obtained a correlation coefficient $r$ $=-0.06$ (between -0.25 and +0.25 ), which means that, in our case, there is a weak correlation between oxygen desaturation and rhinomanometry values.

The dispersion diagram for printing occurs without the dispersing clouds achieving the appearance of an imaginary line that represents linear regression, thus no upward trend. Dependence between oxygen desaturation and the rhinomanometry values is negative, meaning that an increase in oxygen desaturation does not involve an increase in rhinomanometry values (Chart 7).

The results of nasal endoscopy, flexible pharyngoscopy or even of pharyngoscopy during sleeping are subjective results. These examination methods are based on the endoscopic experience of the examiner. Therefore, true statistical correlation between the objective endoscopic exam results and other examination methods considered to be objective could not be made. Flexible pharyngoscopy is considered a subjective method, as there are no dimensional standards of evaluation.

But what should be noted is that flexible pharyngoscopy, as an examination method, must be interpreted in the context of polygraphic and polysomnographic evaluation and not independently. Correlation between endoscopy and polygraphy or polysomnography is crucial in assessing patients with obstructive sleep respiratory pathology.

\section{DISCUSSIONS}

The aim of this study was to present our experience and the role of rhinomanometry, endoscopic and polysomnographic investigations in patients with obstructive sleep apnea syndrome; we were interested to see if the results revealed by the polysomnographic recordings can be correlated with the data found by the methods mentioned above.

Our study reported a statistically significant difference between women and men $(p=0.0001)$, that demonstrates the same aspect as the data from literature, which claim that obstructive sleep apnea is estimated to affect more men than women.

From the literature we know that obesity is the main risk factor for obstructive sleep apnea syndrome, approximately $70 \%$ of patients with obstructive sleep

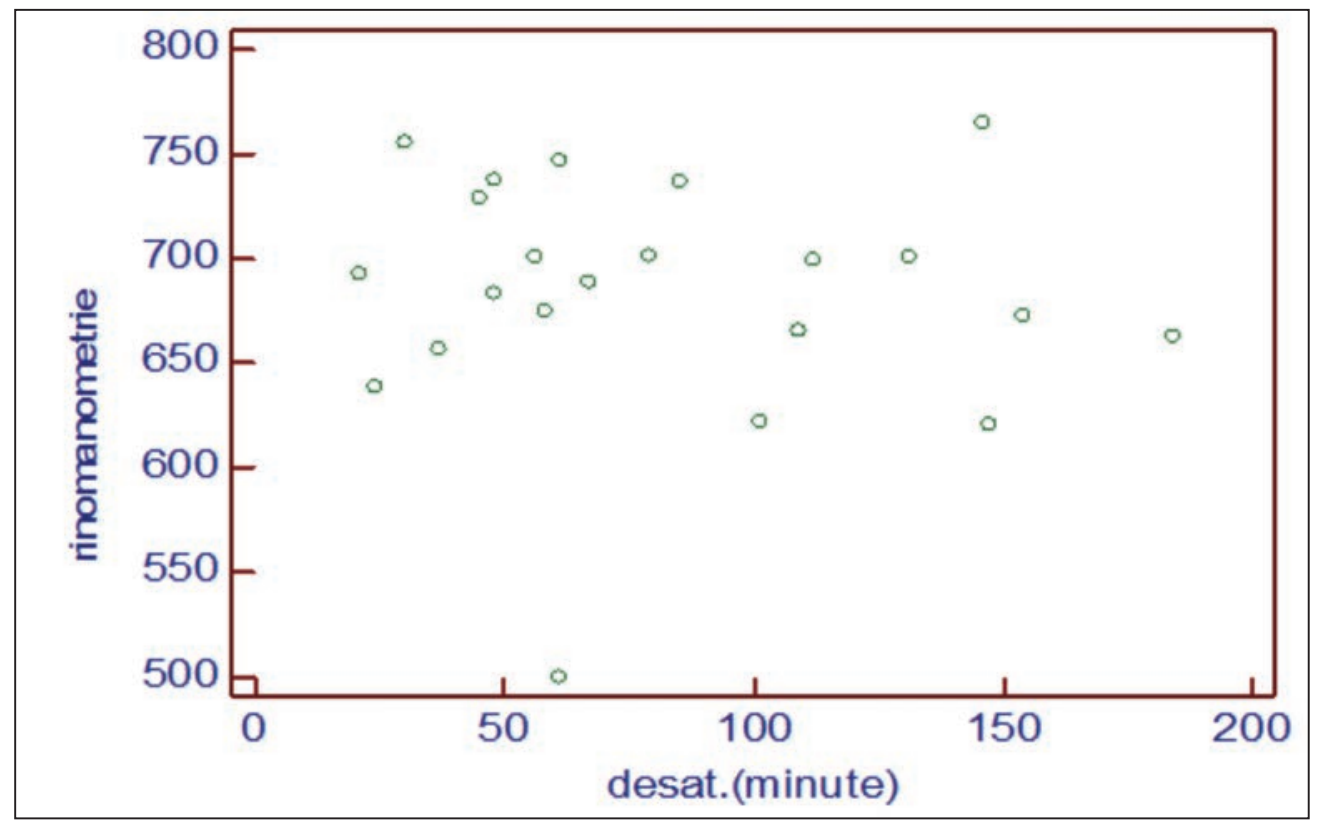

Chart 7 Relation between posterior rhinomanometry and oxygen desaturation 
apnea being obese. So, we considered necessary to determine the body mass index (BMI) in all patients, the average value being of $31.20 \pm 5.09 \mathrm{~kg} / \mathrm{m}^{2}$. This result can be compared to some studies which have reported that the severity of obstructive sleep apnea syndrome in patients with a body mass index of 30 or higher is due to obesity ${ }^{1}$.

Most patients had an apnea-hypopnea index less than $10(41.93 \%$ of the subjects fall in the class of the snoring ones), $48.38 \%$ had the AHI value between 10 50 , with a slight and easy obstructive sleep apnea syndrome and $9.67 \%$ had AHI> 50, so a severe obstructive sleep apnea syndrome. Regarding the changes in the upper airways of patients with OSAS, we did not find a statistically significant difference between nasal changes and AHI severity, with a value of $p$ between 0.25 and 0.75 . However, between the AHI value and nasal allergy $(p=0.06)$, tongue base $(p=0.05)$, the semi-elliptical/omega epiglottis shape $(p=0.05)$, we found a statistically significant relationship. Many studies argue that a deviated nasal septum, nasal polyps and nasal allergy are the most common changes involved in determining the severity of $\mathrm{OSAS}^{7-9}$. There is epidemiological data that takes into account the direct relationship between nasal congestion and obstructive sleep apnea ${ }^{1-3}$.

In the literature, there are numerous studies investigating the patients with obstructive sleep apnea syndrome. Rhinomanometry can be successfully performed in order to calculate nasal resistance, although normal values have never been well established ${ }^{10}$. However, there are conflicting data on the relationship between nasal resistance and the sleepdisordered breathing phenomenon. McNicholas and his colleagues have shown that nasal obstruction due to allergic rhinitis was associated with obstructive sleep apnea ${ }^{11}$. As oronasal breathing was postulated to increase the breathing resistance, there are studies suggesting that, although the degree of resistance is not predictable in nasal obstructive sleep apnea, the presence of an increased nasal resistance can lead from nasal breathing to oronasal breathing, thus increasing the breathing work and further compromising the upper airway ${ }^{12,13}$. In our study, we correlated the results obtained by rhinomanometry investigations with the changes found in the upper airways. Regarding the anatomical change in the nose and the rhinomanometric results, we had no statistically significant relationship between these two parameters. The changes in the size and shape of the tongue base or epiglottis proved to have an important impact upon rhinomanometric values, with a statistically significant value $(p=0.03$, respectively $p=0.08)$.

In terms of the results obtained from the polysomnographic registration, more exactly the value of AHI, there was no statistically significant relationship with the increased nasal resistance values. An explanation for these findings can be the low number of subjects and the lack of information regarding the rhinomanometry investigation. This last aspect is due to the absence of a clear examination protocol in Romania for the patients with sleep pathology.

Endoscopic investigations are needed to locate anatomical changes; however, it is a subjective measurement, even when using classification scores to define the degree of collapse. In addition, the generated negative pressure during the Mueller manoeuvre is variable and it is highly dependent on the patient's effort. Using a manometer can remove this problem, but it will limit the technical availability and it is not routinely recommended. It was suggested that the Mueller manoeuvre can add information to identify the possible positions of obstruction and can predict the patients' outcome after undergoing uvulo-palatopharyngoplasty.

\section{CONCLUSIONS}

Obstructive sleep apnea is more common in middle-aged men, while overweight and also obesity seem to be determining factors. In order to make a proper and correct evaluation of the upper airway changes, the flexible pharyngoscopy is used. It is a subjective measurement and depends on the experience of the examiner, due to which it is supplemented by polysomnography. Polysomnographic recordings are used to detect sleep respiratory pathologies.

Our study demonstrates the existence of a correlation between the rhinomanometric values and the tongue base size and shape of the epiglottis changes. Regarding nasal changes, we found a positive relationship only with nasal allergy. We could not demonstrate the absolute utility and usefulness of rhinomanometry in the assessment of patients with respiratory sleep pathology.

It was demonstrated a correlation between the AHI and nasal allergy, and also with the base of the tongue and the epiglottis shape. So, the results found after endoscopic investigations give significant data on the severity of the obstructive sleep apnea syndrome as the results obtained with polysomnographic registrations.

It is essential that all diagnostic methods to be interpreted in connection with each other.

Conflict of interest: The authors have no conflict of interest.

Contribution of authors: All authors have equally contributed to this work. 


\section{REFERENCES}

1. Georgalas C. - The role of the nose in snoring and obstructive sleep apnoea: an update. Eur Arch Otorhinolaryngol., 2011;268(9):1365-1373. doi: 10.1007/s00405-010-1469-7. Epub 2011 Feb 22.

2. Virkkula P., Bachour A., Hytonen M., Mamlberg H., Salmi T., Maasilta P. Patient and bed partner-reported symptoms, smoking, and nasal resistance in sleep-disordered breathing. Chest., 2005;128(4):2176-2182.

3. Settipane R.A., Charnock D.R. - Epidemiology of rhinitis allergic and nonallergic. Clin Allerrgy Immunol., 2007;19:23-34.

4. Lofaso F., Coste A., d"Ortho M.P., Zerah-Lancner F., Delclaux C., Goldenberg F., Harf A. - Nasal obstruction as a risk factor for sleep apnoea syndrome. Eur Respir J., 2000;16(4):639-643.

5. Miljieteig H., Hoffstein V., Cole P. - The effect of unilateral and bilateral nasal obstruction on snoring and sleep apnea. Laryngoscope., 1992;102(10):11501152 .

6. Miljeteig H., Savard P., Mateika S., Cole P., Haight J.S., Hoffstein V. - Snoring and nasal resistance during sleep. Laryngoscope., 1993;103(8): 918-923.

7. Kushida C.A. - Obstructive Sleep Apnea: Pathophysiology, Comorbidities and Consequences. CRC Press, 2007;p.87-91.
8. Clement P.A., Gordts F., Standardisation Committee on Objective Assessment of the Nasal Airway, IRS, and ERS. - Consensus report on acoustic rhinometry and rhinomanometry. Rhinology., 2005;43(3):169- 179.

9. Hudgel D.W., Hrasick T., Katz R.L., Witt W.J., Abelson T.I. Uvulopalatopharyngoplasty in obstructive apnoea. Value of preoperative localization of site of upper airway narrowing during sleep. Am Rev Respir Dis., 1991;143(5 Pt 1):942-946.

10. Kristo D.A., Lettieri C.J., Andrada T., Taylor Y., Eliasson A.H. - Silent upper airway resistance syndrome: prevalence in a mixed military population. Chest., 2005;127(5):1654-1657.

11. McNicholas W.T., Tarlo S., Cole P., Zamel N., Rutherford R., Griffin D., Phillipson E.A. - Obstructive apneas during sleep in patients with seasonal allergic rhinitis. Am Rev Respir Dis., 1982;126(4):625-628.

12. Oostveen E., Vanderveken O., Verbracken J., et al. - Progressive upper airway narrowing occurs during expiration prior to obstructive, central and mixed apneas. Am J Respir Crit Care Med., 2005;2:608- 610.

13. Rombaux P., Bertrand B., Bodewyns A., et al. - Standard ENT clinical evaluation of the sleep-disordered breathing patient; a consensus report. Acta Otorhinolaryngol Belg., 2002;56(2):127-137. 